\title{
Antibiotics: Past, Present and Future
}

Bushra Jamil ${ }^{1}$ and Muhammad Ali Syed ${ }^{2 *}$

${ }^{1}$ Department of Biosciences, COMSATS Institute of Information Technology, Islamabad, Pakistan

${ }^{2}$ Department of Microbiology, University of Haripur, Haripur, Pakistan

Undoubtedly, antibiotics have brought a revolution to the history of modern medicine and have played a fundamental role in ensuring safe surgical procedures, organ transplants and chemotherapy [1]. Both mortality and morbidity rates of many of infectious diseases have been and morbidity rate have been significantly declined since the introduction of these drugs, since the infectious diseases were always considered as the principal cause of death. Nevertheless, antibiotic's meteoric rise has proved to be short lived, because of rapid emergence of resistance to almost every class of antibiotic [2]. World Health Organization (WHO) has already declared antibiotic resistance among the three paramount threats to health. The theme of World Health Day, on April 7, 2011, was "antimicrobial resistance: no action today and no cure tomorrow" [3]. Such Multi Drug Resistant (MDR) microbes make the treatment more difficult, expensive and associated with more side effects. All those diseases that were under control for long are causing difficulty in their treatment after the emergence of MDR bacteria. The situation continues to be more alarming due to meager efforts put in to develop new drugs [4].

Today, antibiotic resistance is increasing at an alarming rate. Microbes have developed resistance to those antibiotics as well that were considered to be the treatment of last resort. The very recently emerged mechanism of resistance is the secretion of New Delhi metallo $\beta$ lactamase enzymes (NDM). This particular enzyme is prevalent to Indo-Pak region and from this region it is spreading to other parts of world through human travel. Various studies have reported the prevalence of this enzyme in environmental sample as well. This enzyme has the capacity to breakdown carbapenems group of antibiotic. Pertinent to mention here is that carbapenems are the effective and last drugs of choice in curing most of the MDR pathogens. Thus the situation is gruesome and there is a need for more concerted effort [5].

There is a general consensus that nano-antimicrobials (NAMs) could be an effective alternative to conventional antibiotics and helpful in combating drug resistance. Recent studies have reported improved efficacy of antibiotics after nano-encapsulation [6].

Nanotechnology, being considered as the technology of future, is the manipulation of matter on an atomic, molecular or supramolecular scale. Nanotechnology is playing its role in many areas of health sciences, drug and gene delivery, dentistry, and diagnostics, though it's pre-eminent role is considered to be its extraordinary ability to combat resistance [6].

All those nano systems, possessing intrinsic antimicrobial potential or augmenting the overall efficacy and safety of enclosed or adsorbed antibiotics, are termed as "nano-antibiotics. The key advantages of nano-antibiotics over conventional antibiotics are that they can improve bioavailability by enhancing solubility, protecting the drug from premature degradation, both in vivo and during storage. Thus, the desired therapeutic effect could be achieved by improving bioavailability at low dose. Ultimately it will reduce the dose dependent side and toxic effect of drug, and patient compliance will be improved indirectly. By targeted drug delivery, drug will be released at site of action only. The infected site will get the maximum quantity of drug and antimicrobial effect would be optimum. These nano formulations can ensure sustained and controlled release of the drug, which help to reduce therapeutic dose and its frequency. Another advantage offered by nano-antibiotics is their cost effectiveness and stability during manufacturing and shipping. Most important problem associated with conventional antibiotic therapy is antibiotic resistance, which could also be overcome through nano-antibiotics. So the future of conventional antibiotics lies in investing in nano-antibiotics [7].

\section{References}

1. Laxminarayan R, Duse A, Wattal C, Zaidi AK, Wertheim HF et al. (2013) Antibiotic resistance-the need for global solutions. The Lancet Infectious Diseases 13: 1057-1098.

2. Pollack LA, Srinivasan A (2014) Core elements of hospital antibiotic stewardship programs from the Centers for Disease Control and Prevention. Clinical Infectious Diseases 59: S97-S100.

3. Piddock LJ (2012) The crisis of no new antibiotics-what is the way forward? The Lancet Infectious Diseases 12: 249-253.

4. Fair RJ, Tor $Y(2014)$ Antibiotics and bacterial resistance in the $21^{\text {st }}$ century. Perspectives in Medicinal Chemistry 6: 25

5. Nordmann P, Dortet L, Poirel L (2012) Carbapenem resistance in Enterobacteriaceae: here is the storm! Trends in Molecular Medicine 18: 263-272.

6. Jamil B, Bokhari H, Imran M (2015) Mechanism of action: how nanoantimicrobials act? Current Drug Targets 16.

7. Huh AJ, Kwon YJ (2011) "Nanoantibiotics": a new paradigm for treating infectious diseases using nanomaterials in the antibiotics resistant era. Journal of Controlled Release 156: 128-145.
*Corresponding author: Muhammad Ali Syed, Assistant Professor, Department of Microbiology, University of Haripur, Hattar Road, Haripur 22620, Pakistan, Tel: 0092-3325256722; Fax: 0995615394; E-mail: mirwah2000@yahoo.de

Received May 18, 2016; Accepted May 19, 2015; Published May 24, 2016

Citation: Jamil B, Syed MA (2016) Antibiotics: Past, Present and Future. J Biomol Res Ther 5: e149. doi:10.4172/2167-7956.1000e149

Copyright: (c) 2016 Jamil B, et al. This is an open-access article distributed under the terms of the Creative Commons Attribution License, which permits unrestricted use, distribution, and reproduction in any medium, provided the original author and source are credited. 